\title{
Downregulation of the long non-coding RNA taurine-upregulated gene 1 inhibits glioma cell proliferation and invasion and promotes apoptosis
}

\author{
ZHIJUN ZHAO ${ }^{1,2}$, BIN WANG $^{3}$, JUNHAI HAO $^{4}$, WEITAO MAN $^{3}$, YONGKAI CHANG ${ }^{3}$, \\ SHUNCHANG MA ${ }^{3}$, YESHUAI HU ${ }^{3}$, FUSHENG LIU ${ }^{5}$ and JUN YANG ${ }^{2,3}$
}

${ }^{1}$ Department of Neurosurgery, The First Affiliated Hospital of Baotou Medical College, Baotou, Inner Mongolia 014010;
${ }^{2}$ Department of Neurosurgery, Qilu Hospital of Shandong University, Jinan, Shandong 250012; ${ }^{3}$ Department of Neurosurgery,
Fuxing Hospital, Capital Medical University, Beijing 100038; ${ }^{4}$ The Second Clinical Medical College, Southern Medical
University, Guangzhou, Guangdong 510515; ${ }^{5}$ Department of Neurosurgery, Brain Tumor Research Center, Beijing
Neurosurgical Institute, Beijing Tiantan Hospital Affiliated with The Capital Medical University, Beijing 100050, P.R. China

Received April 15, 2016; Accepted November 16, 2017

DOI: $10.3892 / \mathrm{ol} .2018 .7784$

\begin{abstract}
Expression of the long non-coding RNA taurine-upregulated gene 1 (TUG1) is associated with various aggressive tumors. The present study aimed to investigate the biological function of TUG1 in regulating apoptosis, proliferation, invasion and cell cycle distribution in human glioma U251 cells. Lentivirus-mediated TUG1-specific microRNA was transfected into U251 cells to abrogate the expression of TUG1. Flow cytometry analysis was used to examine the cell cycle distribution and apoptosis of U251 cells. Cellular proliferation was examined using Cell Counting Kit-8 (CCK-8) assays and invasion was examined by Transwell assays. The apoptotic rate of cells in the TUG1-knockdown group was significantly higher than in the negative control (NC) group (11.58 vs. 9.14\%, $\mathrm{P}<0.01)$. CCK-8 assay data demonstrated that the proliferative ability of cells within the TUG1-knockdown group was lower compared with that of the NC group. A Transwell invasion assay was performed, which revealed that the number of invaded cells from the TUG1-knockdown group was the less compared with that of the NC group. In addition, the $G_{0} / G_{1}$ phase population was significantly increased within the treated group ( 44.85 vs. $38.45 \%, \mathrm{P}<0.01$ ), as measured by flow cytometry. The present study demonstrated that the downregulation of TUG1 may inhibit proliferation and invasion, and promote glioma U251 cell apoptosis. In addition, knockdown of TUG1 may have an effect on cell cycle arrest.
\end{abstract}

Correspondence to: Dr Jun Yang, Department of Neurosurgery, Qilu Hospital of Shandong University, 107 Wen Hua Xi lu, Lixia, Jinan, Shandong 250012, P.R. China

E-mail: yangjun6778@126.com

Key words: taurine-upregulated gene 1, glioma, apoptosis, proliferation
The data presented in the current study indicated that TUG1 may be a novel therapeutic target for glioma.

\section{Introduction}

Glioma is the most common brain tumor in humans, and is associated with various genetic disorders (1). Patient with glioma continue to have extremely poor prognoses, despite recent advances in chemotherapy, radiotherapy and surgery (1); the median survival in patients with glioblastoma multiforme (World Health Organization, grade IV) may be only $12-15$ months (2).

The non-coding RNAs, including microRNAs (miRNAs) and long non-coding RNAs (IncRNAs), have been demonstrated to serve pivotal functions in the cancer-associated gene regulatory system (3). miRNAs may influence the growth, (3) invasion (4) and apoptosis (5) of glioma by binding with target mRNA sequences. Prior studies have revealed that lncRNAs, non-coding RNAs >200 nucleotides in length, have additionally exhibited tumor regulatory functions (6).

Data from previous studies demonstrated that lncRNAs may function via diverse molecular mechanisms. i) Regulation in the transcription of mRNAs; for example, lncRNA Dlx6 antisense RNA may cooperate with the transcription factor distal-less homeobox 2 (D1x2) to activate the transcription of Dlx5/6 (6). ii) Post-transcriptional regulation of mRNA splicing; for example, the lncRNA zinc finger E-box-binding homeobox 2 (Zeb2) antisense RNA may bind with the 5 '-untranslated region (UTR) intron splice site in the Zeb2 mRNA to prevent splicing of the Zeb2 5'-UTR, increasing the expression levels of Zeb2 protein (7). iii) Epigenetic modifications; for instance, lncRNA HOX transcript antisense RNA (HOTAIR) may recruit polycomb repressive complex 2 to the homeobox D locus, then induce epigenetic silencing at the HOXD locus, resulting in the inhibition of transcription (8).

In addition, Salmena et al (9) proposed the hypothesis of the competing endogenous RNAs in order to explain the function of lncRNAs. The hypothesis states that miRNAs 
may bind to sequences with partial complementarity to target RNAs, known as microRNA response elements (MREs), in order to inhibit the expression of these target RNAs. These MREs exist between mRNAs and lncRNAs, and lncRNAs may competitively bind with certain miRNAs to alleviate the miRNAs-dependent inhibition of mRNA translation. This means that lncRNAs may also influence the expression of coding RNAs via miRNAs (9).

Taurine-upregulated gene 1 (TUG1), a $7.1 \mathrm{~kb}$ 1ncRNA, was first detected via a screening for genes upregulated by taurine in developing mouse retinal cells by Young et al (10). Previous studies have demonstrated that TUG1 expression was upregulated in esophageal squamous cell carcinoma (ESCC), potentially promoting the proliferation of ESCC (11), whereas its expression was downregulated in non-small cell lung carcinoma (NSCLC) (12). However, the function of TUG1 in glioma remains unknown. In the present study, a lentiviral TUG1-interference vector was constructed to downregulate the expression of TUG1 and the impact of TUG1 on glioma cell apoptosis, proliferation, cell cycle and invasion was assessed in vitro.

\section{Materials and methods}

Cell lines and lentiviral infection. The human glioblastoma U251 cell line was purchased from the Cell Bank of Type Culture Collection of Chinese Academy of Sciences (Shanghai, China). The cells were cultured in Dulbecco's modified Eagle's medium (DMEM) containing 10\% fetal bovine serum (FBS; Gibco; Thermo Fisher Scientific, Inc., Waltham, MA, USA) in an atmosphere with $5 \% \mathrm{CO}_{2}$ at $37^{\circ} \mathrm{C}$. For transfection, the cell suspension was seeded into a 6 -well plate at a density of $2 \times 10^{5}$ cells/well for $24 \mathrm{~h}$. The plasmid used was pLenti6.3-MCS/V5 DEST, which was constructed by R\&S Biotechnology Co., Ltd, (Shanghai, China). Then, the cells were transfected with lentivirus-mediated TUG1-miRNA (TUG1-interfered group; treated group) orlentivirus withoutmiRNAs(lenti-EGFP-TUG1-miR-1, $782 \mathrm{ng} / \mathrm{ul}$, OD $(260 / 280)=1.98$; lenti-EGFP-TUG1-miR-2, $890 \mathrm{ng} / \mathrm{ul}$, OD $(260 / 280)=1.99$; lenti-EGFP-TUG1-miR-3, 1,230 ng/ul, OD (260/280)=2.0; lenti-EGFP-TUG1-miR-4, $1,200 \mathrm{ng} / \mathrm{ul}$, OD $(260 / 280)=1.98)$ (negative control group; $\mathrm{NC}$ group; multiplicity of infection=2). The lentiviral vectors without miRNAs and packaging vectors (POLO3000; R\&S Biotechnology Co., Ltd.) were transfected into U251 cells. Half of each group (U251, NC and TUG1-interfered cells) were harvested at $48 \mathrm{~h}$, and the other at $72 \mathrm{~h}$ following transfection, at each time point the infection rate was measured with a fluorescence microscope at magnification, $\mathrm{x} 100$. Excitation at $488 \mathrm{~nm}$ was used for visualization and green fluorescent protein was used). When the infection rate reached $\geq 70 \%, 2 \mathrm{mg} / \mathrm{ml}$ blasticidin (InvivoGen, San Diego, CA, USA) was added into cells for BSD filtering, during which the cells without lentiviral infection were dead. After 6 days, the concentration of blasticidin was reduced to $1 \mathrm{mg} / \mathrm{ml}$, and the stable infected cells (BS-positive) were maintained in culture for additional analysis, in Dulbecco's modified Eagle's medium (DMEM) containing 10\% fetal bovine serum (FBS; Gibco; Thermo Fisher Scientific, Inc., Waltham, MA, USA) in an atmosphere with $5 \% \mathrm{CO}_{2}$ at $37^{\circ} \mathrm{C}$.
Construction of the miRNA lentiviral expression vector. There are four RNA interference candidate target sequences for human lncRNA TUG1, as follows: 5'-TGTGAGCTGTTT CTATGCATA-3'; 5'-CTTTGTTAGTTGTCAGCTGCT-3'; 5'-CTCTTGTTATCAGCCATGGTA-3' and 5'-AGTTCGAAA GCCGCGTCCATT-3'. TUG1-specific miRNA sequences which consisted of artificial RNA for the interference of TUG1, including miTUG1-1 to miTUG1-4, were designed by R\&S Biotechnology, Co., Ltd. All oligonucleotides encoding miRNA sequences were synthesized via polymerase chain reaction (PCR). The thermocycler conditions for the PCR reaction were as follows: Initial pre-denaturation at $95.0^{\circ} \mathrm{C}$ for $3 \mathrm{~min}$, denaturation at $94.0^{\circ} \mathrm{C}$ for $30 \mathrm{sec}$, annealing at $58.0^{\circ} \mathrm{C}$ for $30 \mathrm{sec}$ and final extension at $68^{\circ} \mathrm{C}$ for $1 \mathrm{~min}$, for 30 cycles, using the DNA polymerase Platinum Pfx DNA Polymerase (cat no. C11708021; Invitrogen; Thermo Fisher Scientific, Inc.) and annealed into double strands. The primers used are summarized in Table I (the underlined portions are cutting sites for Enzyme AscI and PmeI). The double-strand RNAs were inserted into pLenti6.3-MCS/V5 DEST lentiviral frame plasmids containing the same cutting sites as the primers (R\&S Biotechnology Co., Ltd.). To screen the target for the most effective inference, transduced U251 cells were collected for quantitative (q)PCR on day 2 following transfection.

\section{Reverse transcription-quantitative polymerase chain reaction} $(R T-q P C R)$. TRIzol ${ }^{\circledR}$ (Life Technologies; Thermo Fisher Scientific, Inc.) was used for cell lysis, following which $130 \mu 1$ chloroform (100\%) and $130 \mu \mathrm{l}$ isopropanol (100\%) were added respectively into lysate, by which high quality total RNA was isolated from the transduced U251 cells. The cDNA kit (Fermentas; Thermo Fisher Scientific, Inc.; cat no. K1622) was used according the protocol of the manufacturer, then RNA was reverse-transcribed to cDNA. qPCR was performed to determine the target with the most effective interference. The RNA primers used in qPCR were as follows: $\beta$-actin forward, 5'-GGCACTCTTCCAGCCTTCC-3', reverse, 5'-GAGCCG CCGATCCACAC-3' and TUG1 forward, 5'-ACGATGCGG CAGGAACAC-3' and reverse, GATGGAATAGCACAG GGAAGG-3. The PCR cycling conditions (40 cycles) were as follows: Pre-denaturation $\left(95.0^{\circ} \mathrm{C}\right.$ for $\left.2 \mathrm{~min}\right)$, denaturation $\left(95.0^{\circ} \mathrm{C}\right.$ for $\left.15 \mathrm{sec}\right)$, annealing $\left(60.0^{\circ} \mathrm{C}\right.$ for $\left.20 \mathrm{sec}\right)$ and extension $\left(72^{\circ} \mathrm{C}\right.$ for $\left.20 \mathrm{sec}\right)$. Then the relative genomic expression was calculated using the $2^{-\Delta \Delta \mathrm{Cq}}$ method (13). Melting curves were generated to ensure that only a single product was amplified. $\beta$-actin was used as an internal control.

Cell proliferation assay. Cell proliferation was assayed using a Cell Counting Kit-8 (R\&S Biotechnology Co., Ltd) (CCK-8) assay. The transfected cells were plated in 96-well plates $\left(2,000\right.$ cells per well, cultured in $5 \% \mathrm{CO}_{2}$ at $\left.37^{\circ} \mathrm{C}\right)$. According to the manufacturer's protocol, cell proliferation was detected at every $24 \mathrm{~h}$ from $0 \mathrm{~h} 5$ consecutive times. Prior to each detection, $10 \mu \mathrm{l}$ of CCK-8 solution was added to each well and incubated for $2 \mathrm{~h}$ at $37^{\circ} \mathrm{C}$. Next, the optical density of each solution was measured at $450 \mathrm{~nm}$.

Cell apoptosis and cell cycle assay. Flow cytometry analysis (using the annexin V-FITC-PI apoptosis analysis kit; R\&S Biotechnology Co., Ltd.) was used to examine the cell 
Table I. Primers for polymerase chain reaction of target genes.

\begin{tabular}{lccc} 
Primers name & Primers sequences & $\begin{array}{c}\text { Temperature, } \\
{ }^{\circ} \mathrm{C}\end{array}$ & $\begin{array}{c}\text { Significance of } \\
\text { underlining }\end{array}$ \\
\hline Lenti-Asc1-F & 5'-TACTGGCGCGCCGCCACCATGGTGAGCAAGGGCGAGGA-3' & 60 & AscI cutting site \\
Lenti-Pme1-R & 5'-ACTAGTTTAAACTGCGGCCAGATCTGGGC-3' & 63 & PmeI cutting site \\
\hline
\end{tabular}

F, forward; R, reverse.

apoptotic rate and the cell cycle following the interference of TUG1. Transfected cells were harvested following transfection and U251 cells were mixed with propidium iodide containing RNase (cat no. R5250; Sigma-Aldrich; Merck $\mathrm{KGaA}$, Darmstadt, Germany), then harvested for $30 \mathrm{~min}$ in $4^{\circ} \mathrm{C}$ without light. The apoptotic rate and cell cycle were examined with a flow cytometer (BD FACSAria Cell Sorter, BD Biosciences, Franklin Lakes, NJ, USA) and BD FACSDiva ${ }^{\mathrm{TM}}$ software v6.1.3 (BD, Biosciences).

Invasion assay. A Transwell assay, with Transwell inserts from Corning Incorporated (corning, NY, USA; cat no. 3422) was performed to investigate the invasiveness of glioma U251 cells. Prior to cell inoculation, $30 \mu 1$ Matrigel was added to each chamber and incubated for $2 \mathrm{~h}$ at $37^{\circ} \mathrm{C}$. Transfected cells were digested by pancreatin to modify the cell concentration to $4 \times 10^{5} / \mathrm{ml}$ and then $0.1 \mathrm{ml}$ of this cell suspension (cells suspended in DMEM with 10\% FBS) was added to the chamber; $0.6 \mathrm{ml}$ complete medium (DMEM with $10 \%$ FBS) was added to the lower plate for $28 \mathrm{~h}$. The chamber was then moved into the plate and cell migration was observed. After $28 \mathrm{~h}$, cells were fixed using $4 \%$ paraformaldehyde (Sigma-Aldrich; Merck KGaA) for $15 \mathrm{~min}$ at room temperature, and the invasion rate and number of cells was analyzed via crystal violet (purchased at a ready-to-use dilution from Sigma-Aldrich, CAS no. 548-62-9) staining at room temperature for 20 min using a light microscope (Olympus BX51/61; Olympus Corporation, Tokyo, Japan).

Statistical analysis. All experiments were performed in triplicate and all data are presented as mean \pm standard error of the mean and were analyzed with SPSS 13.0 statistical software (SPSS, Inc., Chicago, IL, USA). The apoptotic rate, number of cells in the lower chamber and cell numbers in the population of $\mathrm{G}_{0} / \mathrm{G}_{1}$ phase cells were analyzed by one-way analysis of variance to analyze statistical significance followed by Scheffé's post-hoc test. $\mathrm{P}<0.05$ was considered to indicate a statistically significant difference for all statistical analyses.

\section{Results}

Downregulating TUG1 expression in U251 cells via lentiviral vectors transfection. $\mathrm{U} 251$ cells were transfected with lentiviral vectors expressing four different TUGi-targeting miRNAs and the TUG1 expression level was analyzed by qPCR. TUG1-miRNA\#1 was the most effective interfering RNA (Fig. 1). Therefore, the TUG1-interfered miRNA\#1-lentiviral vector was selected for further analysis.

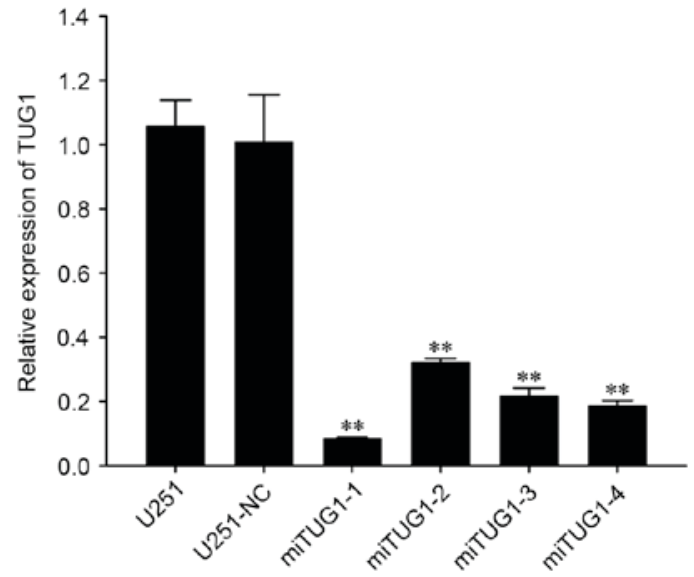

Figure 1. TUG1 expression levels in U251 cells detected by reverse transcription-qPCR. Total RNA from the six types of cells was reverse transcribed and amplified with primers via qPCR. The knockdown efficiency of miRNA\#1, miRNA\#2, miRNA\#3, and miRNA\#4 was 91.6, 68.1, 78.5 and $81.7 \%$, respectively, compared with the negative control. Data are expressed as the mean \pm standard deviation. ${ }^{* *} \mathrm{P}<0.01$ vs. negative control. miRNA, microRNA; NC, negative control; qPCR, quantitative polymerase chain reaction; TUG1, taurine-upregulated gene 1.

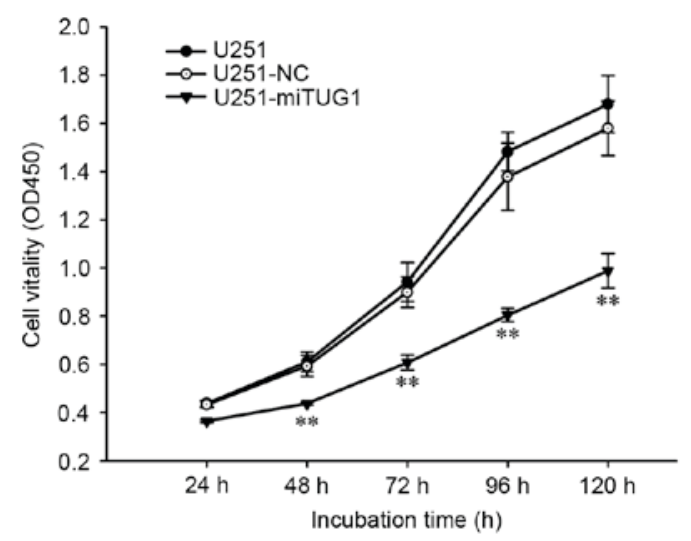

Figure 2. Downregulation of TUG1 inhibits proliferation of U251 cells. Proliferation curves were determined by Cell Counting Kit- 8 assay. Cell growth curves and inhibitory rates of cell growth were generated from OD values obtained at $450 \mathrm{~nm}$. A significant inhibitory effect of cell proliferation was observed from the $48 \mathrm{~h}$ following TUG1 downregulation. Data are expressed as the mean \pm standard deviation. ${ }^{* *} \mathrm{P}<0.01$ vs. negative control. mi, microRNA; NC, negative control; OD, optical density; TUG1, taurine-upregulated gene 1.

Downregulation of TUG1 inhibits cell proliferation in U251 cells. To investigate the function of TUG1 in U251 cell proliferation, a CCK-8 assay was performed and the optical 
A

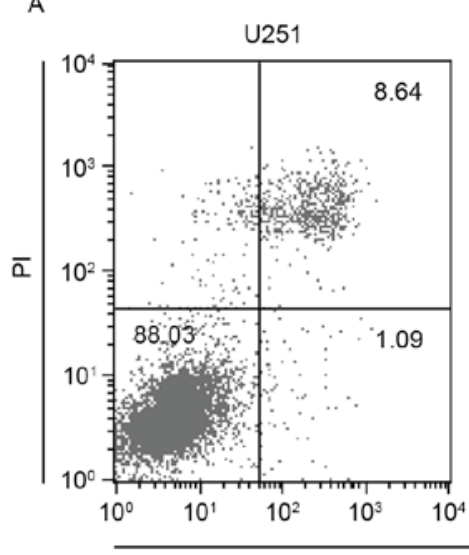

B

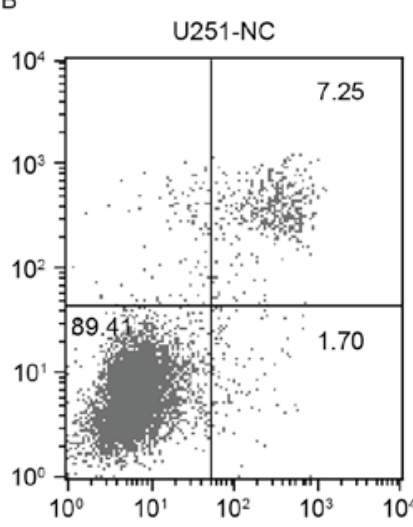

C

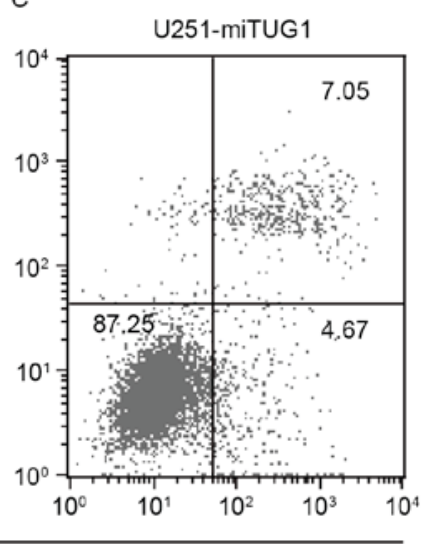

Annexin VIAPC

D

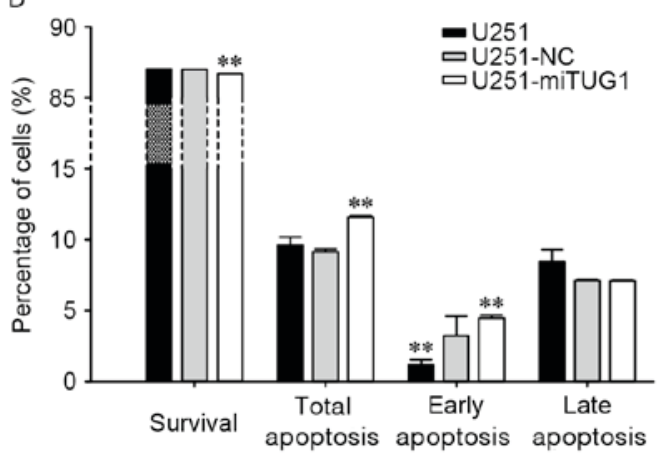

Figure 3. Downregulation of TUG1 promotes apoptosis of U251 cells. Apoptosis of (A) U251, (B) U251-NC (B) and (C) U251-miTUG1 cells was analyzed by flow cytometry. Viable cells were annexin $\mathrm{V}-\mathrm{APC}^{-} / \mathrm{PI}^{-}$(lower left quadrant), dead cells were annexin $\mathrm{V}-\mathrm{APC}^{-} / \mathrm{PI}^{+}$(upper left quadrant), late apoptotic cells were annexin $\mathrm{V}-\mathrm{APC}^{+} / \mathrm{PI}^{+}$(upper right quadrant) and early apoptotic cells were annexin $\mathrm{V}-\mathrm{APC}^{+} / \mathrm{PI}^{-}$(lower right quadrant). (D) The percentage of cells in upper right quadrant and lower right quadrant were totaled to provide the total number of apoptotic cells. The results demonstrated that the downregulation of TUG1 may promote the apoptosis of U251 cells at $48 \mathrm{~h}$, particularly late apoptosis. Data are expressed as the mean \pm standard deviation. ${ }^{* *} \mathrm{P}<0.01 \mathrm{vs}$. negative control. APC, allophycocyanin; mi, microRNA; NC, negative control; OD, optical density; PI, propidium iodide; TUG1, taurine-upregulated gene 1.

density was measured to calculate the cell proliferation. The proliferation of glioma U251 cells was significantly inhibited following the interference of TUG1 compared with that in the negative control group (Fig. 2). The findings of the present study indicated that TUG1 serves a notable role in glioma cell proliferation.

Downregulation of TUG1 promotes cell apoptosis in U251 cells. Flow cytometry analysis was used to examine cell apoptosis and TUG1 function in glioma U251 cell apoptosis. The apoptotic rates between the negative control group and TUG1-interfered group were significantly different (Fig. 3). The apoptotic rate in the U251 cells group without RNA interference was $8.55 \%$ and in the NC group it was $9.14 \%$, whereas in the TUG1-knockdown group it was $11.58 \%(\mathrm{~F}=166.366$; $\mathrm{P}<0.05$; Fig. 3). The findings of the present study indicated that the downregulation of TUG1 expression in glioma cells may significantly promote cell apoptosis.

Downregulation of TUG1 inhibits cell invasion in U251 cells. A Transwell invasion assay was used to assess the invasiveness of U251 cells under different TUG1 expression conditions. The number of cells in the lower chamber was significantly decreased in the TUG1-knockdown group compared with that in the control group (Fig. 4). These findings demonstrated that the downregulation of TUG1 expression may inhibit the invasion of glioma $\mathrm{U} 251$ cells in vitro.

Downregulation of TUG1 induces cell cycle arrest in U251 cells. To investigate the effects of TUG1-knockdown in glioma, the expression of TUG1 was disrupted via lentivirus infection and the cell cycle was analyzed via flow cytometry assay in U251 cells, U251-NC cells, and TUG1-knockdown cells (Fig. 5). The population of $\mathrm{G}_{0} / \mathrm{G}_{1}$ phase cells in the TUG1-knockdown group was significantly increased compared with those in the U251-NC and untransfected U251 groups (44.85 vs. 38.45 vs. $33.25 \%$, respectively; $\mathrm{F}=101.392 ; \mathrm{P}<0.05)$. Conversely, the S-phase fraction was decreased significantly between the TUG1-interfered cells and U251-NC cells (31.76 vs. 35.99 vs. $33.13 \%$, respectively; $\mathrm{F}=5.606 ; \mathrm{P}<0.05)$. As the data revealed, downregulation of TUG1 expression may induce cell cycle arrest at the $G_{0} / G_{1}$ phase in glioma.

\section{Discussion}

The present study demonstrated that the downregulation of TUG1 expression may promote apoptosis in U251 cells and inhibit their proliferation and invasion. TUG1-knockdown has been associated with cell cycle arrest in $G_{0} / G_{1}$ phase (14). Glioma has been reported to be the most common type of 
A

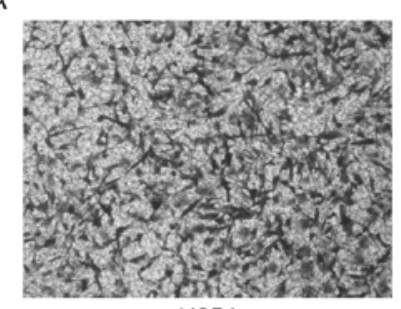

U251
B

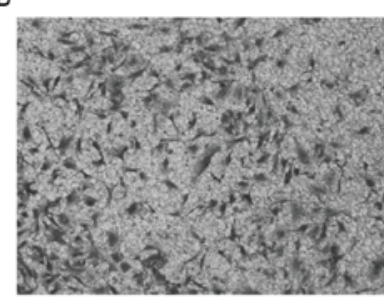

U251-NC
C

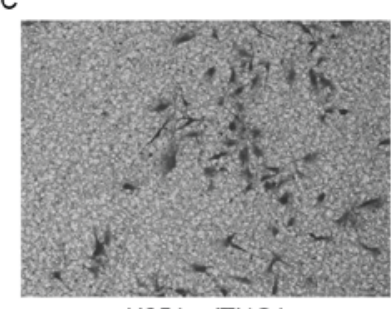

U251-miTUG1

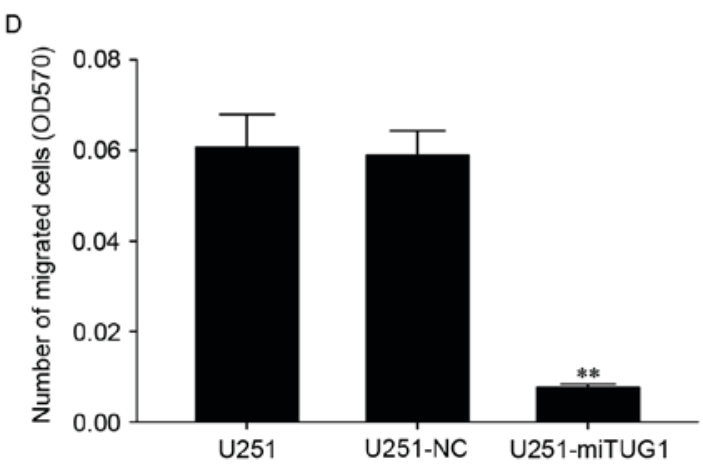

Figure 4. Downregulation of TUG1 impairs the invasive ability of U251 cells. Cells that invaded Matrigel were stained with crystal violet, and the OD570 of the stained cells was calculated to indicate the cell number. Random fields of (A) untransfected U251, (B) NC-transfected U251 and (C) miTUG1-transfected U251 cells were visualized using an inverted microscope (magnification, x40). (D) Histograms visualizing the OD570 of each group. Data are expressed as the mean \pm standard. ${ }^{* *} \mathrm{P}<0.01$ vs. negative control. miTUG1, microRNA targeting taurine-upregulated gene 1; NC, negative control; OD, optical density.

A

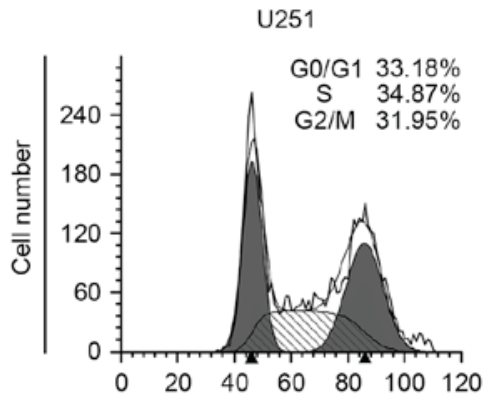

B

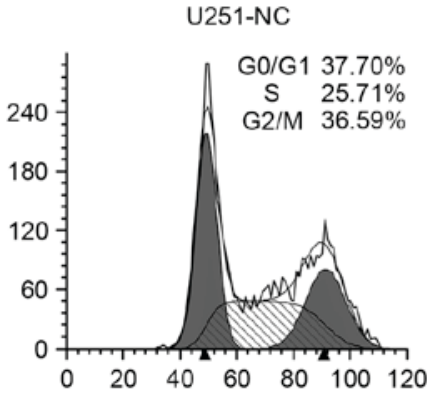

C

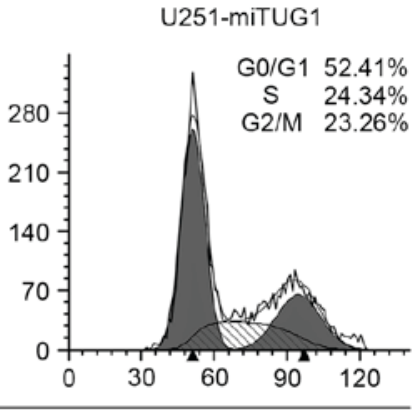

Channels (FL2-A)

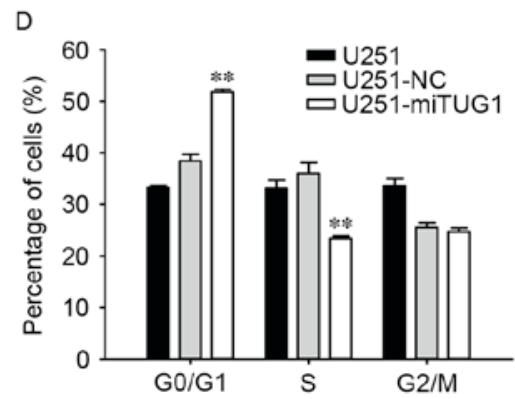

Figure 5. Downregulation of TUG1 triggers cell cycle arrest in glioma cells. (A-C) Results of cell cycle distribution analysis in (A) untransfected, (B) NC-transfected and (C) miTUG1-transfected U251 cells were analyzed by flow cytometry. (D) The percentage of cells in $\mathrm{G}_{0} / \mathrm{G}_{1}, \mathrm{~S}$ and $\mathrm{G}_{2} / \mathrm{M}$ were indicated. U251 cells transfected with miTUG1-1 compared with the negative control demonstrated that the cell cycle was significantly arrested at G0/G1 phase in the TUG1-interfered U251 cells. Data are expressed as the mean \pm standard deviation. ${ }^{* *} \mathrm{P}<0.01$ vs. negative control. miTUG1, microRNA targeting taurine-upregulated gene 1; NC, negative control.

primary cerebral tumor (1). Owing to the highly invasive growth pattern of the disease and the frequent occurrence of resistance to chemotherapy, patients with glioma often succumb to the disease (15). Previous studies $(2,6-7)$ have demonstrated that the mutation of tumor protein $\mathrm{p} 53$, retinoblastoma protein and cyclin-dependent kinase inhibitor $2 \mathrm{~A}$ are involved in the development of glioma; however, the etiology and pathogenesis of glioma remain unclear. Previous studies $(3,5,16)$ have indicated that miRNAs regulate the expression of target mRNAs, which can have an effect on tumor behaviors, particularly glioma. 
Over the past few years, a number of IncRNAs have been identified, including HOTAIR, imprinted maternally expression transcript (H19), metastasis-associated lung adenocarcinoma transcript 1 (MALAT1) and maternally expressed 3 (MEG3) (16). Recent studies have demonstrated that lncRNAs may serve important roles in the genesis and developmental processes of tumors $(5,17,18)$. For instance, Li et al (17) demonstrated that the IncRNA HOXA transcript may promote proliferation, invasion and chemoresistance in pancreatic ductal adenocarcinoma, and may represent a potential therapeutic target. Han et al (18) reported that the IncRNAs expression profiles between glioma and normal brain tissue differed significantly via gene chip technology analysis. LncRNAs HOTAIR (19), H19 (20) and MEG3 (21) have been demonstrated to be associated with glioma, including proliferation, invasion and apoptosis. Additionally, the lncRNA tumor suppressor in lung cancer 1-antisense RNA (22) and MALAT1 (1) may be factors that allow for prognostic prediction.

TUG1 was first identified in the mouse retina and is expressed during retinal development (10). Han et al (23) reported that TUG1 is overexpressed in urothelial carcinoma of the bladder and may be a biomarker of bladder urothelial carcinoma and/or a novel target of gene therapy. Zhang et al (24) revealed that downregulating the expression of TUG1 could inhibit osteosarcoma cell proliferation and promote apoptosis, meaning that TUG1 could represent a novel diagnostic marker and a therapeutic target for patients with osteosarcoma. $\mathrm{Xu}$ et al (11) demonstrated that upregulating TUG1 expression could promote cell proliferation and migration in esophageal squamous cell carcinoma. However, the association between TUG1 and glioma requires further investigation.

Liu et al (25) assessed the association between glioma and numerous lncRNAs, including TUG1, and demonstrated that lncRNAs may be involved in the process of cellular defense against genotoxic agents; however, the mechanism by which TUG1 affects the biological processes of glioma has not been reported. To the best of our knowledge, the present study is the first to investigate the function of TUG1 in glioma.

In the present study, a lentivirus containing a TUG1-targeting miRNA was used to transfect U251 cells, downregulating TUG1 expression. The invasive ability of U251 cells was assessed using a transwell invasion assay, whereas cellular proliferation was assayed using a CCK-8 assay. In addition, flow cytometry analysis was performed to measure cell cycle distribution and apoptotic rate of cells. Together, these data revealed that TUG1 expression levels might affect the biological progresses of glioma, including apoptosis, proliferation and invasion. The Transwell invasion assay demonstrated that TUG1 expression was associated with the invasion of U251 cells and the invasiveness was inhibited markedly following TUG1-knockdown. The CCK- 8 assay revealed that proliferation was significantly inhibited by the interference of TUG1. In addition, the apoptotic rate of cells in the TUG1-knockdown group was significantly lower than that of cells in the control group, which indicated that TUG1 might regulate the apoptosis of glioma. Furthermore, the effects of TUG1 interference on the cell cycle in U251 cells were investigated. The results of the present study revealed that the downregulation of TUG1 induced cell cycle arrest at the $\mathrm{G}_{0} / \mathrm{G}_{1}$ phase. Collectively, the present study demonstrated that
TUG1 may be associated with numerous biological processes in glioma and may serve a role in certain oncogenic processes.

Zhang et al (12) discussed the association between TUG1 expression and NSCLC, revealing that TUG1 expression was downregulated in NSCLC and associated with poor prognosis. TUG1 may be an important factor in the p53-regulatory network; p53 is a tumor suppressor gene, the mutation of which is associated with the occurrence and progression of numerous cancer types, including those of the liver (26), breast (27), bladder (28) and stomach (29). As reported, p53 may also serve an important function in the occurrence and development of brain glioma (30). Previous studies have reported that p53 is involved in cell cycle arrest, promotion of apoptosis and maintenance of genomic stability $(2,3,8,25)$. The present study revealed that TUG1 may promote cell apoptosis and induce cell cycle arrest, and downregulating TUG1 may function as a p53 downstream effector. Therefore, TUG1 may be an important factor associated with p53 and glioma; however, further investigation is required.

In conclusion, the results of the present study revealed that downregulation of the IncRNA TUG1 may promote glioma cell apoptosis, and inhibit proliferation and invasion in vitro. Knockdown of TUG1 may induce cell cycle arrest in $\mathrm{G}_{0} / \mathrm{G}_{1}$ phase. Thus, the downregulation of TUG1 may represent a novel strategy for gene therapy for glioma.

\section{Acknowledgements}

The present study was supported by the Inner Mongolia Natural Science Foundation (grant no. 2015MS0859), the Young Scientists Foundation of Fu Xing Hospital of Capital Medical University (grant no. 2014YQ) and the National Natural Science Foundation of China (grant nos. 81372354, 81302186 and 81271563).

\section{References}

1. Ma KX, Wang HJ, Li XR, Li T, Su G, Yang P and Wu JW: Long noncoding RNA MALAT1 associates with the malignant status and poor prognosis in glioma. Tumour Biol 36: 3355-3359, 2015.

2. Grossman SA, Ye X, Piantadosi S, Desideri S, Nabors LB, Rosenfeld M and Fisher J; NABTT CNS Consortium: Survival of patients with newly diagnosed glioblastoma treated with radiation and temozolomide in research studies in the United States. Clin Cancer Res 16: 2443-2449, 2010.

3. Guo M, Zhang X, Wang G, Sun J, Jiang Z, Khadarian K, Yu S, Zhao Y, Xie C, Zhang K, et al: miR-603 promotes glioma cell growth via wnt/ $\beta$-catenin pathway by inhibiting WIF1 and CTNNBIP1. Cancer Lett 360: 76-86, 2015.

4. Gu JJ, Gao GZ and Zhang SM: miR-218 inhibits the migration and invasion of glioma U87 cells through the Slit2-Robo1 pathway. Oncol Lett 9: 1561-1566, 2015.

5. Shang C, Hong Y, Guo Y, Liu YH and Xue YX: MiR-210 up-regulation inhibits proliferation and induces apoptosis in glioma cells by targeting SIN3A. Med Sci Monit 20: 2571-2577, 2014.

6. Feng J, Bi C, Clark BS, Mady R, Shah P and Kohtz JD: The Evf-2 noncoding RNA is transcribed from the Dlx-5/6 ultraconserved region and functions as a Dlx-2 transcriptional coactivator. Gnens Dev 20: 1470-1484, 2006.

7. Beltran M, Puig I, Peña C, García JM, Alvarez AB, Peña R, Bonilla $F$ and de Herreros AG: A natural antisense transcript regulates Zeb2/Sip1 gene expression during Snail1-induced epithelial-mesenchymal transition. Genes Dev 22: 756-769, 2008.

8. Rinn JL, Kertesz M, Wang JK, Squazzo SL, Xu X, Brugmann SA, Goodnough LH, Helms JA, Farnham PJ, Segal E and Chang HY: Functional demarcation of active and silent chromatin domains in human HOX loci by noncoding RNAs. Cell 129: 1311-1323, 2007. 
9. Salmena L, Poliseno L, Tay Y, Kats L and Pandolfi PP: A ceRNA hypothesis: The rosetta Stone of a hidden RNA language? Cell 146: 353-358, 2011.

10. Young TL, Matsuda T and Cepko CL: The noncoding RNA taurine upregulated gene 1 is required for differentiation of the murine retina. Curr Biol 15: 501-512, 2005.

11. Xu Y, Wang J, Qiu M, Xu L, Li M, Jiang F, Yin R and Xu L: Upregulation of the long noncoding RNA TUG1 promotes proliferation and migration of esophageal squamous cell carcinoma. Tumour Biol 36: 1643-1651, 2015.

12. Zhang EB, Yin DD, Sun M, Kong R, Liu XH, You LH, Han L, Xia R, Wang KM, Yang JS, et al: P53-regulated long non-coding RNA TUG1 affects cell proliferation in human non-small cell lung cancer, partly through epigenetically regulating HOXB7 expression. Cell Death Dis 5: e1243, 2014.

13. Livak KJ and Schmittgen TD: Analysis of relative gene expression data using real-time quantitative PCR and the 2(-Delta Delta C(T)) method. Methods 25: 402-408, 2001

14. Yan G, Wang X, Yang M, Lu L and Zhou Q: Long non-coding RNA TUG1 promotes progression of oral squamous cell carcinoma through upregulating FMNL2 by sponging miR-219. Am J Cancer Res 7: 1899-1912, 2017.

15. Cuddapah VA, Robel S, Watkins S and Sontheimer H: A neurocentric perspective on glioma invasion. Nat Rev Neurosci 15 $455-465,2014$

16. Zhang K, Sun X, Zhou X, Han L, Chen L, Shi Z, Zhang A, Ye M, Wang Q, Liu C, et al: Long non-coding RNA HOTAIR promotes glioblastoma cell cycle progression in an EZH2 dependent manner. Oncotarget 6: 537-546, 2015.

17. Li Z, Zhao X, Zhou Y, Liu Y, Zhou Q, Ye H, Wang Y, Zeng J, Song Y, Gao W, et al: The long non-coding RNA HOTTIP promotes progression and gemcitabine resistance by regulating HOXA13 in pancreatic cancer. J Transl Med 13: 84, 2015.

18. Han L, Zhang K, Shi Z, Zhang J, Zhu J, Zhu S, Zhang A, Jia Z, Wang G, Yu S, et al: LncRNA profile of glioblastoma reveals the potential role of lncRNAs in contributing to glioblastoma pathogenesis. Int J Oncol 40: 2004-2012, 2012.

19. Zhang JX, Han L, Bao ZS, Wang YY, Chen LY, Yan W, Yu SZ, Pu PY, Liu N, You YP, et al: HOTAIR, a cell cycle-associated long noncoding RNA and a strong predictor of survival, is preferentially expressed in classical and mesenchymal glioma. Neuro Oncol 15: 1595-1603, 2013.
20. Shi Y, Wang Y, Luan W, Wang P, Tao T, Zhang J, Qian J, Liu N and You Y: Long non-coding RNA H19 promotes glioma cell invasion by deriving miR-675. PLoS One 9: e86295, 2014.

21. Wang $P, \operatorname{Ren} Z$ and Sun P: Overexpression of the long non-coding RNA MEG3 impairs in vitro glioma cell proliferation. J Cell Biochem 113: 1868-1874, 2012.

22. Qin X, Yao J, Geng P, Fu X, Xue J and Zhang Z: LncRNA TSLC1-AS1 is a novel tumor suppressor in glioma. Int J Clin Exp Pathol 7: 3065-3072, 2014.

23. Han Y, Liu Y, Gui Y and Cai Z: Long intergenic noncoding RNA TUG1 is overexpressed in urothelial carcinoma of the bladder. J Surg Oncol 107: 555-559, 2013.

24. Zhang Q, Geng PL, Yin P, Wang XL, Jia JP and Yao J: Down-regulation of long non-coding RNA TUG1 inhibits osteosarcoma cell proliferation and promotes apoptosis. Asian Pac J Cancer Prev 14: 2311-2315, 2013.

25. Liu Q, Sun S, Yu W, Jiang J, Zhuo F, Qiu G, Xu S and Jiang X: Altered expression of long non-coding RNAs during genotoxic stress-induced cell death in human glioma cells. J Neurooncol 122: 283-292, 2015.

26. Jiang L, Zhang Q, Ren H, Ma S, Lu C, Liu B, Liu J, Liang J, Li M and Zhu R: Dihydromyricetin enhances the chemo-sensitivity of nedaplatin via regulation of the $\mathrm{p} 53 / \mathrm{Bcl}-2$ pathway in hepatocellular carcinoma cells. PLoS One 10: e0124994, 2015.

27. Shafiee SM, Rasti M, Seghatoleslam A, Azimi T and Owji AA: UBE2Q1 in a human breast carcinoma cell line: Overexpression and interaction with p53. Asian Pac J Cancer Prev 16: 3723-3727, 2015.

28. Lee K, Jung ES, Choi YJ, Lee KY and Lee A: Expression of $\mathrm{pRb}, \mathrm{p} 53, \mathrm{p} 16$ and cyclin D1 and their clinical implications in urothelial carcinoma. J Korean Med Sci 25: 1449-1455, 2010.

29. Yildirim M, Kaya V, Demirpence O, Gunduz S and Bozcuk H: Prognosis significance of 553 in gastric cancer: A meta-analysis. Asian Pac J Cancer Prev 16: 327-332, 2015.

30. Elhag R, Mazzio EA and Soliman KF: The effect of silibinin in enhancing toxicity of temozolomide and etoposide in p53 and PTEN-mutated resistant glioma cell lines. Anticancer Res 35: 1263-1269, 2015 